\title{
Modelos estratégicos para el diseño de la historia clínica electrónica
}

\begin{abstract}
$E_{\mathrm{n}}$
un momento en el que la informatización de registros clínicos es ya un hecho más o menos cotidiano en las diversas administraciones con competencias sanitarias de nuestro país, puede parecer extemporáneo el referirse a posiciones estratégicas -aquellas que deberían soportar el trasfondo del hecho mismo de informatizar- en la propia concepción de la historia clínica electrónica.

Y, sin embargo, la situación ha llegado a un punto que el obviar por más tiempo analizar en público los pros y contras de estos modelos, puede conducir a un callejón de difícil salida, si la decisión que se tome en este campo no es acertada o es tomada, por los responsables de hacerlo, por razones diferentes a las estrictamente sanitarias, como parece que está sucediendo a tenor de la facilidad con que se descalifican modelos que, curiosamente, soportan la base de los desarrollos en el mundo occidental (USA, Canadá, Reino Unido, Francia, Holanda, Bélgica, Suecia, Finlandia, etc.).

Pretendemos, por tanto, mostrar la trascendencia e implicaciones de las diferentes vías de abordaje estratégico de los registros clínicos que hoy día están generando inversiones milmillonarias por parte de administraciones sanitarias de nuestro país.
\end{abstract}

\section{MODELOS PARA EL DISEÑO DE UNA HISTORIA CLÍNICA INFORMATIZADA}

Al pensar en la historia clínica informatizada ideal de uno de nuestros pacientes, la imagen que normalmente se nos viene a la cabeza es la de un único historial completo del mismo, que contenga todas aquellas intervenciones que se han producido sobre él en cualquier parte y en cualquier momento. De la misma forma, al pensar en las historias clínicas de la población general extendemos el concepto mentalmente a una si tuación que permita que lo anteriormente descrito sea extensible a cualquier paciente que pueda solicitar asistencia, ahora o en un futuro, y en cualquier parte.

Esta supuestamente idílica visión, factible en realidad desde hace más de diez años, no ha sido llevada a la práctica más que en contadas y controvertidas situaciones, generalmente en el seno de corporaciones sanitarias privadas de pago por servicio, chocando tanto con las cada vez más frecuentes negativas de pacientes y profesionales a que dicha información se encuentre en un formato de esa naturaleza, como con la 
tozuda realidad de un mundo en el que prima la competencia, la libertad de pensamiento y el derecho a la privacidad.

La posibilidad de incorporar de forma ágil desarrollos nuevos, diferentes y personalizables, que den rápida respuesta a necesidades intrínsecas del sistema (administración) o de cada uno de sus actores en su medio (profesionales sanitarios, no sanitarios y población), se encontraría gravemente comprometida en un entorno que, como el descrito, ha de plantearse como un desarrollo monolítico, vertical e impuesto.

De otra parte, la disponibilidad de información para la toma de decisiones, está comenzando a chocar, entre otros, con el derecho a la privacidad de los ciudadanos como individuos. Dicha información está siendo vivida por éstos cada vez más como una amenaza, por su impacto social, laboral, sobre hábitos de consumo, etc., generando frecuentes conflictos y, en países que nos llevan delantera, denuncias por parte de quienes se sienten indefensos ante una tecnología creada teóricamente para su beneficio y de cuyos usos finales comienzan a desconfiar.

Conviene por tanto definir ahora algunos conceptos, en principio contrapuestos, aunque su resultado final de cara a la existencia y manejo de información puede ser idéntico, y que constituyen la base de un desarrollo diferente de las historias clínicas informatizadas, con trascendencia para alguno de los aspectos comentados:

\section{Historia centralizada versus historia descentralizada}

El concepto hace referencia a la localización física de los datos. En el caso de las historias centralizadas, éstos se localizan en uno o varios macroservidores que almacenan todos los datos sanitarios de toda la población a la que se ofrece cobertura, por ejemplo 3 millones de habitantes, independientemente del nivel asistencial desde el que se acceda y, ocasionalmente incluso, del proveedor que preste los servicios. Estos datos han de ser gestionados siempre a través de una red informatizada en cualquier momento que se precisen o depositen y, por tanto, su circulación es siempre continua a pesar de que su uso sea innecesario para el fin sanitario que se persigue.

Por el contrario, en el caso de la historia descentralizada, los datos se encuentran distribuidos en diferentes pequeños, o no tan pequeños, servidores localizados preferentemente en los propios establecimientos sanitarios (kospitales, centros de salud...), que almacenan la información de las personas adscritas o contactadas por los mismos y transfieren, mediante red informática, sólo aquellos que son precisos a otros niveles previamente fijados en formatos también prefijados, como pueden ser informes de alta o interconsultas, resultados de pruebas o imágenes; o cualquier otro tipo de datos o formatos cuando le son específicamente requeridos (links de personas autorizadas), entre los que se incluye la 
creación de historias electrónicas virtuales autocompuestas en servidores seguros de las intranets o internet.

Los defensores de la centralización aducen que con su sistema la información está disponible, por definición, al completo. Las medidas de seguridad centralizada dificultarían el robo-daño de la información y, en cualquier caso, si éste se produce sería consecuencia de un delito del que no se sienten responsables, pues cumplen con las normas de seguridad, y es a la ley a quien corresponde su persecución.

Sus detractores opinan que el montaje inicial es más caro, al alcance como proveedores casi exclusivo de las grandes corporaciones vendedoras de hardware, lo que produce una limitación de competencia por exclusión. El mantenimiento, hoy día, con las posibilidades de gestión remota de software, y las nuevas formas de adquisición de hardware que impone la propia obsolescencia de los equipos (p. ej. renting con mantenimiento incluido) tampoco es ya más barato. Cuantitativamente la mayor parte de la información almacenada es útil e interpretable exclusivamente por quien la almacena (un nivel asistencial, un servicio, un profesional concreto), de forma que en el caso de la Atención Primaria más del 95\% de la información generada es útil exclusivamente para ese nivel.

En este sentido, otro aspecto a considerar sería el elevado número de transacciones y el volumen de información que tendría que soportar un servidor central. Todos los puestos médicos, de enfermería, administrativos, etc., de Atención Primaria, más los de Atención Especializada, interactuando simultáneamente en la misma base de datos, supondrían una sobrecarga casi insoportable para las redes actualmente instaladas, obligando a la contratación de nuevas redes de gran capacidad y por tanto de alto coste.

Las averías centrales, por otra parte no infrecuentes, desconectan a todo el sistema haciendo que la dependencia tecnológica sea absoluta.

Los peligros para la sociedad son enormes, ante robo o pérdida, de forma que si la base es difundida una única vez no hay reparación posible. Si bien, para un profano, la diferencia puede parecer sutil, desde el punto de vista de la seguridad y confidencialidad, la opción de historia descentralizada es más respetuosa con los derechos individuales, pues la información, estando igualmente disponible gracias a la red cuándo, dónde y por quién se necesite, no puede ser violada al completo desde un único punto, de forma que, si no es necesario centralizar ¿por qué arriesgarse?, y si no es necesario enviar toda la información ¿por qué hacerlo?, ya que en este punto se cumple el axioma que el valor de la información crece exponencialmente con su volumen (conviene señalar aquí que en la mayor parte de las denuncias producidas por difusión de datos personales se encuentra imputado personal interno de la gestión de las propias bases). Es necesario tener en cuenta que las medidas de seguridad para evitar accesos incontrolados a la base de datos, no sólo 
se centran en la securización de estas bases, sino también en los accesos a la red (protegida por "cortafuegos"), la cual puede ser violada independientemente de la localización física de los datos (ya sea en una base centralizada o distribuida).

Otro aspecto que suele olvidarse porque, en nuestro entorno, no "parece" formar parte de la actividad central de los clínicos, es el uso de los registros como fuente de investigación. En el caso de la historia centralizada, para el diseño de instrumentos de investigación clínica nos veremos obligados bien a incorporar un departamento central de diseño que elabore los protocolos y pantallas que den soporte a las bases de datos que precisen los estudios, o bien a incorporar herramientas de diseño local que parcelarían el entorno central que nos habíamos marcado, y por tanto éstas cuando existen tienden exclusivamente a permitir diseños restrictivos. Y si la cuestión es grave con el diseño de los estudios, ¿qué decir de su evaluación de resultados? El modelo central debería soportar la interrogación de sus bases de datos por todos aquellos que estén inmersos en proyectos de investigación para extraer tanto la información de casos como la de controles, de forma que, incluso en un medio tan parco en estos como el de nuestro país, la saturación e incomodidad sería evidente, tanto para los investigadores como para todo el sistema que se vería sobrecargado y ralentizado en sus respuestas. El modelo de historia descentralizada, por su propia concepción no padece de ninguno de estos extremos, pues los programas incorporan sus propias herramientas locales de diseño e interrogación de sus bases de datos internas, limitando el fenómeno al propio centro o servicio del que parte la iniciativa. En relación con posibles estudios multicéntricos, el único problema existente en las bases de datos descentralizadas es la necesidad de interrogar a cada una de ellas por separado previamente a la integración de la información, problema en realidad menor en comparación con el esfuerzo de planificación, coordinación, normalización de procedimientos, verificación y control que precisan este tipo de estudios.

En cualquier caso, dado el desarrollo tecnológico actual, el problema no sería tanto responder a la pregunta: ¿cuán segura y eficiente es la historia clínica centralizada?, como a una pregunta anterior: ¿para qué es necesaria?, porque si la respuesta es que para el manejo de los problemas de los pacientes no aporta beneficios tangibles, los riesgos de su implantación son evidentes, y a los profesionales puede dificultarles diferentes parcelas de su práctica: ¿a quién beneficia su uso?

\section{Historia única versus historia integrada}

Historia única es aquella cuyo formato es compartido por los diferentes profesionales sanitarios que tienen acceso a los datos de un paciente, aun cuando existan restricciones de visualización según el tipo de usuario. 
Historia integrada es aquella en la cual el formato de la misma para los diferentes profesionales se encuentra adaptado a su forma/entorno de trabajo (clasificaciones diferentes, estructura distinta...), estando el nexo común constituido básicamente por la llamada Información No Redundante (INR) -aquella que recogida una sola vez está presente en todos los puntos que puedan necesitarla- pactada y consensuadamente incremental, del sistema sanitario al que presten servicio, pudiéndose rescatar el resto en caso de que quien lo solicite esté autorizado a hacerlo, con las consiguientes claves de acceso e identificación en cada nivel.

La necesidad de los clínicos de disponer de forma ideal de un formato "unificado" se confunde con el desarrollo por los informáticos de una única historia, cuando la realidad cotidiana demuestra que esta forma de trabajo ni existe ni es eficiente. Consideremos por ejemplo a quien, cada vez que abre una historia, salvo al dermatólogo que lo controla, le interesa disponer, por defecto, de las 40 fotografías del seguimiento de un liquen plano; a quien salvo al internista que lo siguió los 20 controles de hematocrito en la recuperación de un sangrado digestivo; a quien, salvo al traumatólogo que la realizó, la película de una artroscopia; o a quien, salvo al profesional responsable de hacerlo, el nivel de cumplimentación en un paciente de las normas técnicas de una cartera de servicios de Atención Primaria, por poner algunos ejemplos de información que hoy día ya contienen historias clínicas informatizadas.

En el caso concreto de las clasificaciones de enfermedad, la evidencia demuestra que las necesidades de Atención Primaria se centran en la sensibilidad y la prevalencia, mientras que, en el caso de Atención Especializada, éstas se centran en la especificidad diagnóstica. Este hecho sutil provoca que sea mucho más eficiente utilizar codificaciones específicas para cada nivel, como repetidamente indican la OMS, la WONCA y todos los estudiosos del tema, de forma que sea factible etiquetar los problemas en base a sus necesidades de respuesta, evitando innecesarios fondos de saco de imposible manejo.

De otra parte, la codificación en Atención Primaria se produce en tiempo real, por el clínico encargado del proceso, mientras que en Atención Especializada son generalmente documentalistas quienes se encargan a posteriori del etiquetado de los mismos, parece por tanto lógico ofrecer a aquéllos las máximas facilidades para el manejo de la incertidumbre que genera su práctica.

Otro aspecto a considerar es la gestión de procesos, el comúnmente llamado ciclo general del pedido en las empresas de servicios. Cuando se aboga por la existencia de formatos únicos, se desconoce, u obvia, que la historia clínica informatizada no es una fotografía o sucesión estática de fotografías; la historia electrónica es, más bien, un formato interactivo donde las escenas -pasos dados para la resolución de procesos- pueden concatenarse o dispararse secuencialmente en función del argumento cambiante -el tipo de problema- que nos es presentado. Es 
evidente para cualquiera que cada nivel sanitario, cada problema, cada situación geográfica, cada dotación estructural, condicionan grandemente la cadena de este ciclo y, si esto es así, ¿cómo se soporta el mismo a través de un entorno secuenciado idéntico?

Un formato de historia único, finalmente, al igual que ocurría con la centralización, afecta también de forma negativa a la investigación clínica, pues dificulta extraordinariamente la recopilación de información en formularios específicos a tal efecto, de forma que el clínico debe acudir a diferentes partes de la misma para estar seguro de cumplimentar todos los items que tiene comprometidos en su estudio, recurriendo con frecuencia a introducir información en formatos no procesables (datos en modo texto).

\section{Historia conectada versus historia no conectada}

Prácticamente todos los nuevos desarrollos ofrecen la posibilidad de conexión, a través de una red informática, de las historias clínicas. Sin embargo, aún persisten algunos profesionales reticentes con la conexión a otros puestos diferentes al del clínico aislado que recaba la información. El único aspecto positivo de este tipo de desarrollos está constituido por la mejora en la seguridad de los datos, mientras que, por otro lado, minimizan hasta tal punto las ventajas de la informatización que esta opción está ampliamente desprestigiada como solución estratégica.

Las conexiones entre diferentes ordenadores a través de una red informática permiten el intercambio ágil de información que, de otra forma, sería prácticamente estanca. El paradigma de este hecho es la red de Internet, en la que diferentes bases de información descentralizada, repartidas por todo el mundo, se comportan de cara al usuario como una única base central, argumento que, por sí mismo, ya prueba la eficiencia y posibilidad de manejo descentralizado de datos en todos los órdenes del conocimiento.

El desarrollo de las redes corporativas de comunicación permite que las bases de datos descentralizadas se comporten en realidad como si físicamente se encontrasen en un único punto, o sea fuesen una base centralizada, aportando con respecto a ésta las ventajas ya expuestas en aspectos de confidencialidad del ciudadano y libertad de diseño para el usuario interno. $\mathrm{Si}$ a este entorno, le aportamos las herramientas de securización hoy día disponibles, mantenimiento remoto y necesaria formación del usuario en la trascendencia de su manejo correcto, podemos afirmar sin dudas que es la solución estratégica más conveniente, y desde luego la más difundida entre los países que nos llevan delantera en este terreno tecnológico.

Como apunte filosófico final hay que resaltar que la conexión es un hecho físico, mientras que la comunicación es una actitud intelectual. De nada vale poseer redes de comunicación si no inculcamos o facilitamos a los profesionales las actitudes y mecanismos necesarios para que las conexiones trasladen el contenido cualitativamente correcto. 
En las actuales circunstancias, analizando el desarrollo en el ámbito mundial y las perspectivas que enmarcan tanto la legislación actual de nuestro entorno como las recomendaciones de diferentes grupos de trabajo, y sus propuestas de futuro, parece que el modelo más eficiente, respetuoso, realista y que ofrezca ventajas tanto para la administración sanitaria de nuestro sistema sanitario público como para la práctica del profesional sanitario, y para la defensa de los intereses reales, y no argumentos espurios, de la población, es aquel que provee de una historia clínica integrada, descentralizada e interconectada, por otra parte asumido sistemáticamente en la implantación de desarrollos informáticos, por la práctica totalidad de países con los que compartimos modelos políticos, económicos o sociales.

F. A. Alonso López* y J. Custodi i Canosa

*Doctor en Medicina y Cirugía Especialistas en Medicina Familiar y Comunitaria INSALUD - Subdirección General de Atención Primaria

\section{BIBLIOGRAFÍA}

1. Alonso López FA, et al. Informatización en la Atención Primaria. Documento semFYC no 13. Barcelona. Sociedad Española de Medicina Familiar y Comunitaria, 1999.

2. Evaluation of GP Computer Systems. National Report. NHS Executive. http://ctf.imc.exec.nhs.uk/cards/c0000351.htm (documento integro en internet)

3. Kibbe D, Bard MR. How safe are Computerized Patient records? Family Practice Management 1997; 4:5.

4. California Healthcare Foundation. Promoting Health. Protecting Privacy: A Primer. Los Angeles: California Healthcare Foundation and Consumers Union, 1999.

5. Rehms S, Kraft S. Electronic Medical Records: The FPM Vendor Survey. Family Practice Management 2001; 8:45-54.

6. Burton Community: Pilot Electronic Health Record 1998-1999, Final Overview Report. http://www.schin.ncl.ac.uk/mig/burton.pdf (documento íntegro en internet).

7. Good Practice Guidelines for General Practice Electronic Patient Records. The Joint Computing Group of the General Practitioners' Comittee and the Royal College of General Practitioners. Londres: The NHS Executive General and Personal Medical Services Branch, 2000.

8. Alonso López FA, Ruiz Téllez A, Guijarro Eguskizaga A, Oyarzabal Camio JI, Pérez Gortari P, Aldanondo Gabilondo A. La hipertrofia benigna de próstata en la clínica de Atención Primaria. Estudio multicéntrico de prevalencia diagnóstica, perfil de los pacientes, farmacoterapia y costes derivados. Madrid: GlaxoWellcome, 2000 (documento íntegro en en Internet http://www.institutoapcom.org). 